\title{
THE INFLUENCE OF RUTHENIUM AND RHENIUM ON THE LOCAL PROPERTIES OF THE $\gamma$-AND $\gamma$-PHASE IN NICKEL-BASE SUPERALLOYS AND THEIR CONSEQUENCES FOR ALLOY BEHAVIOR
}

\author{
S. Neumeier, F. Pyczak, M. Göken \\ University Erlangen-Nürnberg, Institute General Materials Properties, Erlangen, Germany
}

Keywords: ruthenium, rhenium, partitioning behavior, reverse partitioning, lattice constants, nanoindentation, creep

\begin{abstract}
Rhenium and ruthenium are alloyed to modern nickel-base superalloys to gain good high temperature properties combined with acceptable phase stability. Three experimental alloys with varied contents of rhenium and ruthenium were investigated. It was found with transmission electron microscopy (TEM)-based energy dispersive spectroscopy (EDS) that rhenium distributes more homogenously between the $\gamma$ - and $\gamma$-phase in the presence of ruthenium (reverse partitioning). Other properties of the alloys as the lattice misfit, local hardness of the individual phases and the high temperature evolution of the microstructure were strongly influenced by the partitioning behavior of rhenium and ruthenium. The changes in lattice constants and hardness of the $\gamma$ - and $\gamma^{\prime}$ phase could be verified by X-ray diffraction and nanoindentation in an atomic force microscope (NI-AFM). The optimum $\gamma^{\prime}$-size with respect to creep properties was found to be dependent on the lattice misfit.
\end{abstract}

\section{Introduction}

Nickel-base superalloys today are alloyed with more than ten alloying elements to achieve e. g. enhanced oxidation resistance or solid solution strengthening of the matrix phase [1]. It is difficult to obtain an exact understanding of the role of each individual element since a number of alloy properties are altered. Rhenium for example is usually alloyed due to its solid solution hardening effects. But rhenium also changes the lattice misfit, hinders $\gamma^{\prime}$-coarsening by slowing down diffusion and sometimes deteriorates the mechanical properties of the alloy by promoting the precipitation of brittle topologically close packed phases (TCP-phases).

To overcome the problem with reduced phase stability in rhenium containing alloys ruthenium was added. Nevertheless, while the finding that ruthenium improves the phase stability is experimentally well documented, the reason for this is not clear $[2,3]$. Especially an effect of so called reverse partitioning, i.e. that rhenium is less strongly enriched in the $\gamma$-matrix if ruthenium is present and accordingly the critical concentration of rhenium in the matrix to precipitate TCP-phases is not reached, was found by O'Hara et al first [3] but could not be reproduced by a number of other groups investigating ruthenium containing alloys $[4,5]$. Newer results explain these discrepancies in reverse partitioning not only by the presence or absence of ruthenium but also by the presence and content of other alloying elements. Especially chromium and cobalt are of importance in this respect [6].

As for reverse partitioning, also the influence of ruthenium on other alloy properties, e.g. solid solution strengthening [7, 8], needs further investigation. Nevertheless, ruthenium containing alloys are very interesting with respect to application as these alloys have the highest creep strength at temperatures in the $1100{ }^{\circ} \mathrm{C}$ range known today [7]. As any element possibly influences a number of alloy properties only investigations employing different characterization methods can try to draw a complete picture. This gets even more complicated as the effects of alloying elements can be different for the $\gamma$ - and $\gamma^{\prime}$-phase and hence local investigation methods are necessary to measure the properties of these two phases independently.

In the present work we characterize the influence of rhenium and ruthenium additions in nickel-base superalloys by investigating experimental alloys with systematically varied rhenium and ruthenium content. To gain information on the changes in different alloy properties analytical transmission electron microscopy (TEM), X-ray diffraction and atomic force microscopy (AFM) combined with nanoindentation (NI-AFM) were employed.

The alloys were investigated in standard heat treated state, after high temperature annealing and after creep experiments.

\section{Experimental}

The three experimental alloys investigated here have the same base composition besides the rhenium and ruthenium content. The nominal composition of the alloys is given in table 1 . The alloys were directionally solidified in a Bridgman unit as rods of $8 \mathrm{~cm}$ length and $2.4 \mathrm{~cm}$ diameter. All three alloys were solution heat treated to fully dissolve the coarse cast $\gamma$-structure and eutectic regions. The solution heat treatment times were chosen sufficiently long to fully homogenize the distribution of alloying elements between dendrite core and interdendritic region. Subsequently the alloys were age hardened for $4 \mathrm{~h}$ at $1100^{\circ} \mathrm{C}$ and $24 \mathrm{~h}$ at $850^{\circ} \mathrm{C}$ (from now on termed as standard heat treated state). To generate coarsened microstructures some specimens were heat treated at $1100{ }^{\circ} \mathrm{C}$ up to $750 \mathrm{~h}$.

To obtain different $\gamma^{\prime}$-sizes the heat treatment step at $1100{ }^{\circ} \mathrm{C}$ was extended for up to $24 \mathrm{~h}$ with subsequent ageing for additional $24 \mathrm{~h}$ at $850^{\circ} \mathrm{C}$ as in the standard heat treatment. A heat treatment at $1100{ }^{\circ} \mathrm{C}$ lasting $24 \mathrm{~h}$ was used for the specimens investigated with nanoindentation to coarsen the precipitates and increase the channel width, which makes it easier to indent the $\gamma$ - and $\gamma^{\prime}$-phase separately.

Generally, for all kinds of microstructural investigations the specimens were ground and subsequently polished with diamond suspension. For standard metallographic investigations in a light and scanning electron microscope (SEM) the specimens were additionally etched with an agent, which preferentially removed the $\gamma^{\prime}$-phase. To gain a slight height contrasts between $\gamma$ and $\gamma^{\prime}$ phase in the AFM the specimens were additionally chemo- 
Table 1: Nominal composition of the three alloys investigated in this work.

\begin{tabular}{|l|c|c|c|c|c|c|c|c|c|}
\hline Element/wt.\% & Al & Ti & Cr & Co & Mo & Ta & Re & Ru & Ni \\
\hline Alloy ReRu & 4.9 & 3.9 & 8.2 & 4.1 & 2.5 & 1.6 & $\mathbf{3 . 0}$ & $\mathbf{3 . 0}$ & 68.8 \\
\hline Alloy Re & 4.9 & 3.9 & 8.2 & 4.1 & 2.5 & 1.6 & $\mathbf{3 . 0}$ & 0.0 & 71.8 \\
\hline Alloy 0 & 4.9 & 3.9 & 8.2 & 4.1 & 2.5 & 1.6 & 0.0 & 0.0 & 74.8 \\
\hline
\end{tabular}

mechanically polished with nanodisperse $\mathrm{SiO}_{2}$ suspension in a vibrational polishing machine. This preparation method leads to a very smooth specimen surface with a small height difference between the $\gamma$-matrix and the $\gamma$-precipitates of only some nanometers. For specimens for X-ray investigations no $\gamma$-phase selective etching was performed. TEM-specimens were electrolytically thinned using an agent of perchloric and acetic acid in a mixing ration of 1:10.

In the investigations a Jeol 6400 SEM and a Philips CM200T TEM with an EDAX-System for energy dispersive spectroscopy (EDS) was used. X-ray diffraction measurements were performed in a dual crystal diffraction setup with negligible instrumental peak broadening according to Wilkens and Eckert [9]. To derive the lattice constants of the $\gamma$ - and $\gamma$-phase from the measured Xray profiles the measured peak profiles were split up in two or three sub-profiles. This was done by combining up to three Pseudo-Voigt peak profiles in such a way that the added intensity of the Pseudo Voigt profiles reassembled the measured overall Xray profile. The sub-profiles were identified as the contributions of the $\gamma$-phase and the $\gamma$-phase (in the matrix channels perpendicular and parallel to the diffraction plane if three subprofiles were used to fit the overall X-ray profile). This was done using the XOP software package [10]. With this X-ray setup, lattice constants can be measured with an accuracy of about $\pm 0.0002 \mathrm{~nm}$. The error in lattice misfit measurements is in the order of $0.01 \%$.

The nanoindentation investigations were performed at room temperature with a Triboscope force transducer from Hysitron mounted on a Veeco Instruments Multimode AFM (NI-AFM). For scanning as well as indentation a diamond tip with cube corner geometry (a three-sided pyramidal indenter) was used. In the indentation measurements a maximum load of $250 \mu \mathrm{N}$ was applied. This resulted in a maximum indentation depth of about $45 \mathrm{~nm}$. This ensured that both phases could be indented separately. AFM images of the three experimental alloys including nanoindents are shown in Fig. 1. The nanohardness of $\gamma$ and $\gamma$ were calculated from the unloading part of the loaddisplacement curves recorded during indenting by using the Oliver/Pharr method [11]. At least 10 indents for each phase were evaluated.

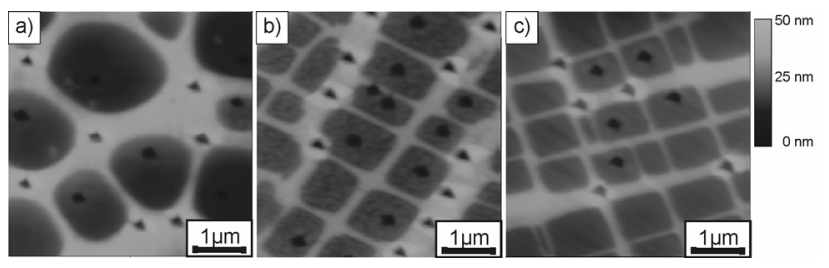

Figure 1: AFM-micrographs with nanoindents in a) Alloy 0 b) Alloy Re and c) Alloy ReRu.

Annealing experiments were done in air. A sufficiently thick surface layer has been removed during subsequent specimen preparation to remove oxidized or chemically depleted zones. All creep tests were performed under compression at air with cylindrical specimens of $7.5 \mathrm{~mm}$ length and $5 \mathrm{~mm}$ diameter. The temperature at the specimen surface was controlled by a thermocouple with an accuracy of $+/-3 \mathrm{~K}$. The test temperature was $1100{ }^{\circ} \mathrm{C}$ and a stress of $137 \mathrm{MPa}$ was applied. Extended ageing heat treatments were also performed in air at $1100{ }^{\circ} \mathrm{C}$ for up to $750 \mathrm{~h}$. To measure the distribution of alloying elements between $\gamma$ - and $\gamma^{\prime}$-phase at high temperature by TEM/EDS a similar procedure as already used by Hemmersmeier et al [12] was performed. These specimens were heated up to the temperature of interest in the range between 1150 and $1300{ }^{\circ} \mathrm{C}$ held for 60 minutes at this temperature and then quenched in water by opening a shutter and allowing the specimens to fall directly from the furnace chamber to a water bath to achieve high cooling rates. In this work the results obtained for a temperature of $1150{ }^{\circ} \mathrm{C}$ are shown and discussed.

\section{Results}

\section{Microstructure}

The microstructures of the three alloys in the standard heat treated condition do not exhibit any TCP-phases. This phase stability is also retained after ageing at $1100{ }^{\circ} \mathrm{C}$ for up to $750 \mathrm{~h}$. Nevertheless, the $\gamma / \gamma$-microstructures encountered in the three alloys show strong differences. As visible in Fig. 2 the shape of the $\gamma$-precipitates is rather globular in Alloy 0 while a more cubic shape is found in Alloy Re and Alloy ReRu exhibits near perfect $\gamma^{\prime}$-cubes. The $\gamma^{\prime}$-particle size is the highest in Alloy 0 , intermediate in Alloy Re and the smallest in Alloy ReRu (see also Fig. 3).
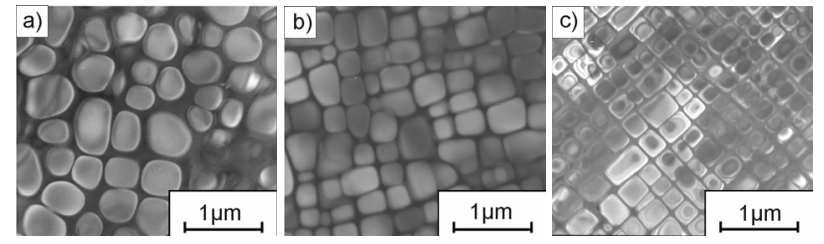

Figure 2: TEM dark field images of the microstructure of a) Alloy 0, b) Alloy Re and c) Alloy ReRu.

\section{Microstructure Development during Annealing}

The microstructure of the alloys was also investigated after annealing. During short time annealing up to $24 \mathrm{~h}$ the $\gamma^{\prime}$ precipitates coarsen isotropically in all three alloys. Nevertheless, the rate of coarsening is different in the three alloys. The evolution of the average precipitate size $a$ is shown in Fig. 3, where $(a / 2)^{3}$ is plotted versus annealing time. The precipitate growth is much faster in Alloy 0 compared to both rheniumcontaining alloy variants. The coarsening rate in Alloy Re is slightly faster than in Alloy ReRu. SEM-micrographs of the three 
alloys after annealing at $1100{ }^{\circ} \mathrm{C}$ for $750 \mathrm{~h}$ are shown in Fig. 4 . While the $\gamma^{\prime}$-precipitates in Alloy 0 have coarsened isotropically and developed a slightly cubic shape the morphology of the $\gamma$ precipitates in Alloy Re and Alloy ReRu is different. In these two alloys the $\gamma$-precipitates coarsened directionally. Elongated particles grow along two preferred directions which are perpendicular to each other and parallel to the crystallographic $<100>$ orientations.

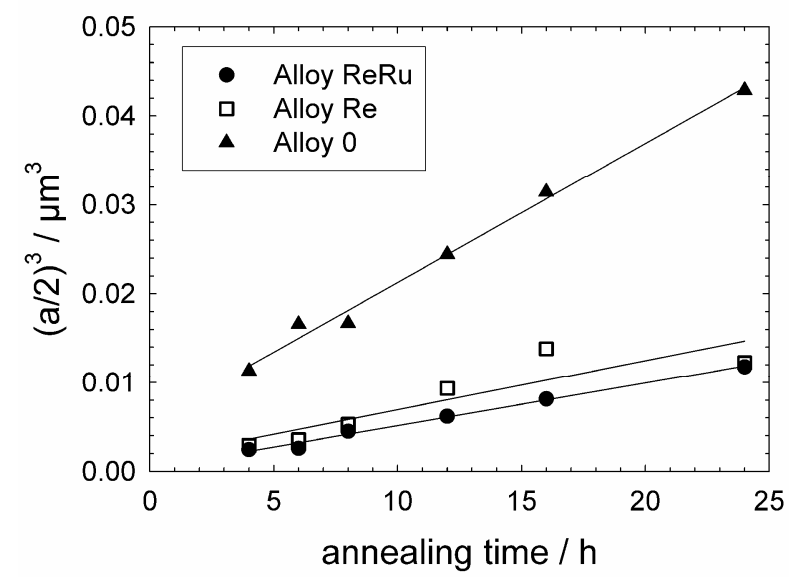

Figure 3: Evolution of the $\gamma^{\prime}$-particle size $a$ of the experimental alloys during short-time annealing at $1100{ }^{\circ} \mathrm{C}$.

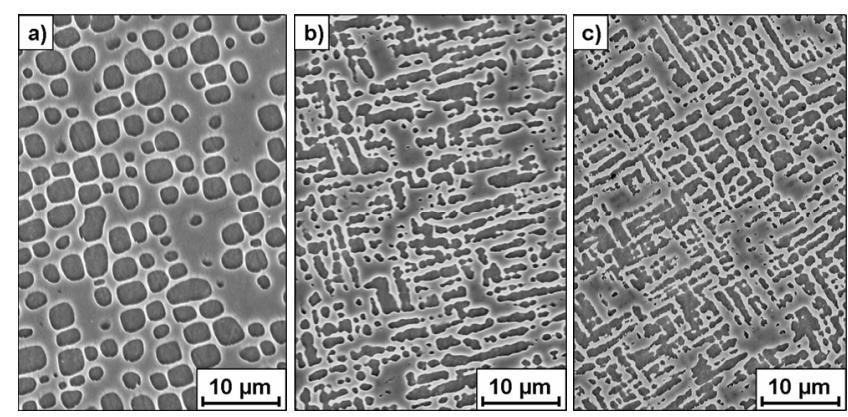

Figure 4: SEM-micrographs of a) Alloy 0, b) Alloy Re and c) Alloy ReRu after annealing at $1100{ }^{\circ} \mathrm{C}$ for $750 \mathrm{~h}$.

\section{Partitioning Behavior}

From the local chemical composition of the $\gamma$-matrix and the $\gamma$ particles measured by EDS in the TEM the partitioning coefficient for the different alloying elements was calculated. In the following the partitioning coefficient $k$ is used

$$
k_{i}^{\gamma^{\prime} / \gamma}=\frac{c_{i}^{\gamma^{\prime}}}{c_{i}^{\gamma}}
$$

with $c_{i}^{\gamma^{\prime}}$ being the concentration of alloying element $i$ in the $\gamma$ and $c_{i}^{\gamma}$ in the $\gamma$-phase, respectively. A k-coefficient greater than one denotes that an alloying element is preferentially enriched in the $\gamma$-precipitates. The k-coefficients for the three alloys in the standard heat treated condition are plotted in Fig. 5. Tantalum is not shown, due to the small overall content of tantalum in the alloys. Most alloying elements show the partitioning behavior known from literature $[4,5,6,13,14,15]$. This is also true for ruthenium which is found to be slightly enriched in the $\gamma$-phase. Rhenium has the strongest tendency to partition to the $\gamma$-matrix compared with the other alloying elements in Alloy Re. Rhenium and to a lesser extent also molybdenum partitions more homogenously if ruthenium is added as in Alloy ReRu. So reverse partitioning of rhenium is found in the presence of ruthenium in the alloys investigated in this work. The $\gamma^{\prime}$-volume fraction was also determined from the partitioning coefficients and the overall alloy composition by Blavette's method [16]. The highest volume fraction with $73 \%$ is found in Alloy ReRu and the lowest volume fraction with $65 \%$ in Alloy 0 . Alloy Re has a $\gamma^{\prime}$-volume fraction of $71 \%$.

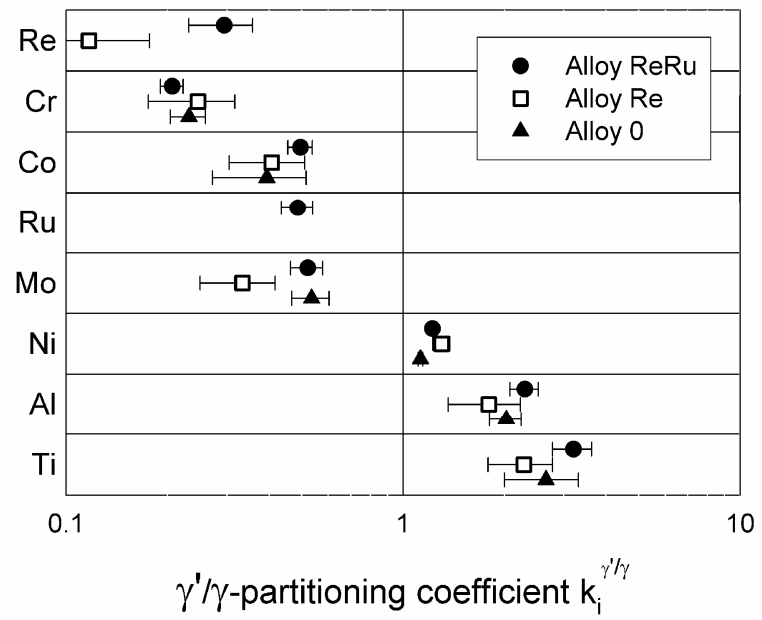

Figure 5: Partitioning coefficients of the alloying elements in the standard heat treated condition of Alloy 0, Alloy Re and Alloy ReRu.

The k-coefficients of the alloying elements at $1150{ }^{\circ} \mathrm{C}$ measured in specimens after rapid quenching as described in the experimental section are plotted in Fig. 6.

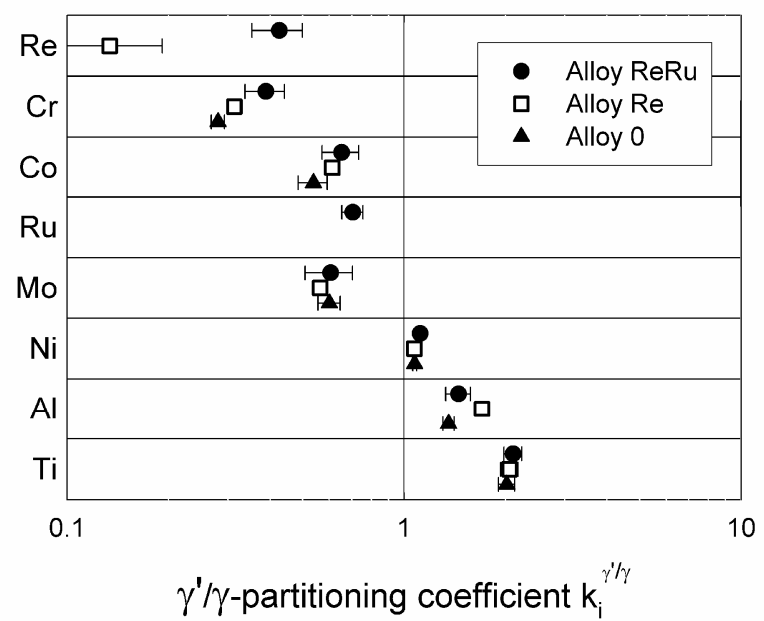

Figure 6: Partitioning coefficients of the alloying elements at $1150{ }^{\circ} \mathrm{C}$ in Alloy 0, Alloy Re and Alloy ReRu.

It is obvious that all alloying elements distribute more homogenously between $\gamma$ - and $\gamma^{\prime}$-phase at higher temperatures. Two of the results are of special interest with respect to the 
behavior of rhenium and ruthenium: Ruthenium which has a tendency to enrich in the $\gamma$-matrix as pronounced as cobalt or molybdenum in the standard heat treated state homogenizes slightly faster with increasing temperature than molybdenum and cobalt and the effect of reverse partitioning measured in the standard heat treated state of Alloy ReRu is also present at higher temperatures.

\section{$\underline{\text { Lattice Constants and Lattice Misfit }}$}

In Fig. 7 the X-ray profiles of the (002)-reflection for the three alloys in standard heat treated condition are shown. The profiles of Alloy 0 and Alloy ReRu have a pronounced asymmetric shape. It is commonly accepted that this results from the overlap of the sub-peaks of the $\gamma$ - and $\gamma^{\prime}$-phase which possess slightly different lattice constants. Nevertheless, while the asymmetry in the case of Alloy 0 is on the side of smaller lattice constants, Alloy ReRu exhibits an asymmetry at higher lattice constant side.

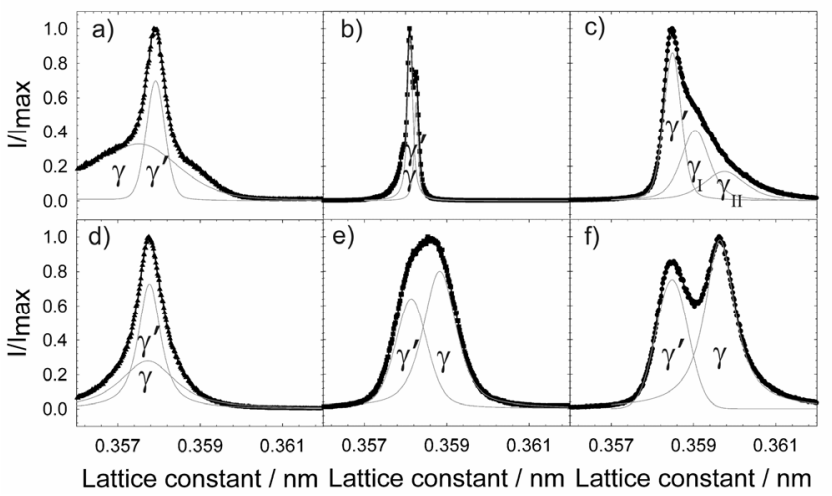

Figure 7: (002)-X-ray profiles of a) Alloy 0, b) Alloy Re and c) Alloy ReRu in the standard heat treated state and of d) Alloy 0, e) Alloy Re and f) Alloy ReRu after compressive creep at $1100^{\circ} \mathrm{C}$.

The lattice misfit $\delta$ is commonly defined in nickel-base superalloys as

$$
\delta=\frac{2\left(a_{\gamma^{\prime}}-a_{\gamma}\right)}{a_{\gamma^{\prime}}+a_{\gamma}}
$$

with $a_{\gamma}$ and $a_{\gamma}$ being the lattice constants of $\gamma$ - and $\gamma$-phase, respectively. This means that the lattice misfit is positive in Alloy 0 and negative in Alloy ReRu. Alloy Re in the standard heat treated condition has a very symmetric X-ray profile indicating a lattice mismatch value near zero.

The lattice constant of the $\gamma$-phase is increasing from Alloy 0 with $0.3575 \mathrm{~nm}$ over Alloy Re with $0.3583 \mathrm{~nm}$ to Alloy ReRu with $0.3593 \mathrm{~nm}$. The lattice constant of the $\gamma$-phase does not change significantly between Alloy 0 with $0.3579 \mathrm{~nm}$ and Alloy Re with $0.3581 \mathrm{~nm}$ while it increases in Alloy ReRu to $0.3585 \mathrm{~nm}$.

These changes in the lattice constants of the $\gamma$ - and $\gamma^{\prime}$-phase result in according changes of the lattice misfit. In Fig. 8 the lattice misfit is plotted vs. the added content of the elements rhenium and ruthenium in the alloys. For the standard heat treated states the lattice misfit decreases linearly with increasing rhenium and ruthenium content. Besides the standard heat treated states X-ray diffraction measurements were also performed on specimens crept at $1100{ }^{\circ} \mathrm{C}$ under a compressive load of $137 \mathrm{MPa}$. The creep tests were interrupted at a plastic strain of $6.5 \%$ (Alloy ReRu and
Alloy 0) and $11.5 \%$ (Alloy Re), respectively. The diffraction measurements were done on (002)-lattice planes under reflection, which lay perpendicular to the external stress axis. The (002)-peak of the crept specimen of Alloy 0 is more symmetric compared to the standard heat treated state as the lattice constants of the $\gamma$ - and $\gamma$-phase after creep are less different. Due to creep deformation the lattice constant of the $\gamma$-matrix increased and the lattice misfit changed to near zero. An increasing peak broadening from the standard heat treated state to the crept state is found in Alloy Re and Alloy ReRu. The separation of the sub-peaks of $\gamma$ - and $\gamma$ is even more pronounced in Alloy ReRu containing a high negative lattice misfit already in the standard heat treated state at room temperature. In Fig. 8 it is visible that the misfit in the crept specimen is always lower than in the standard heat treated state and that also after creep the misfit decreases with increasing content of rhenium and ruthenium in the alloys.

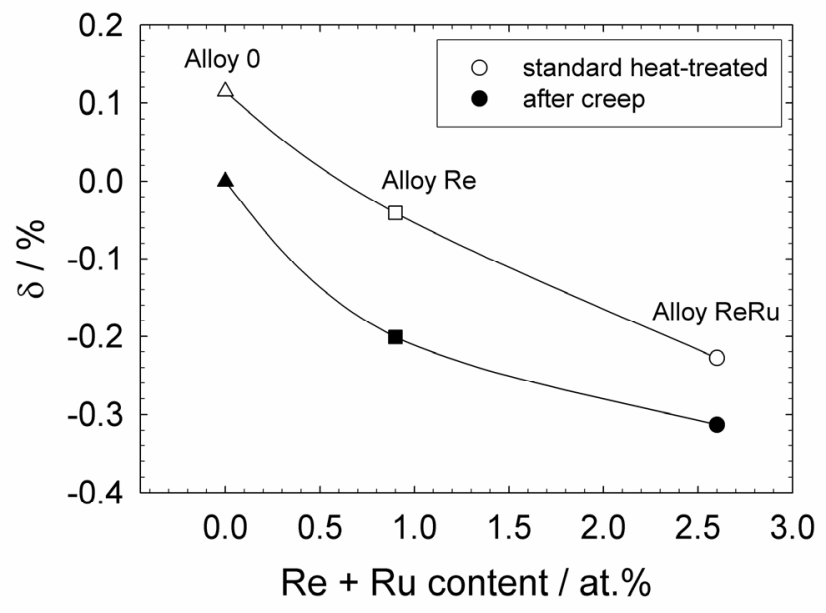

Figure 8: Lattice misfit for the standard heat treated states and specimens crept at $1100^{\circ} \mathrm{C}$ plotted versus the combined content of rhenium and ruthenium.

Hardness of $\gamma$ - and $\gamma^{\prime}$-Phase

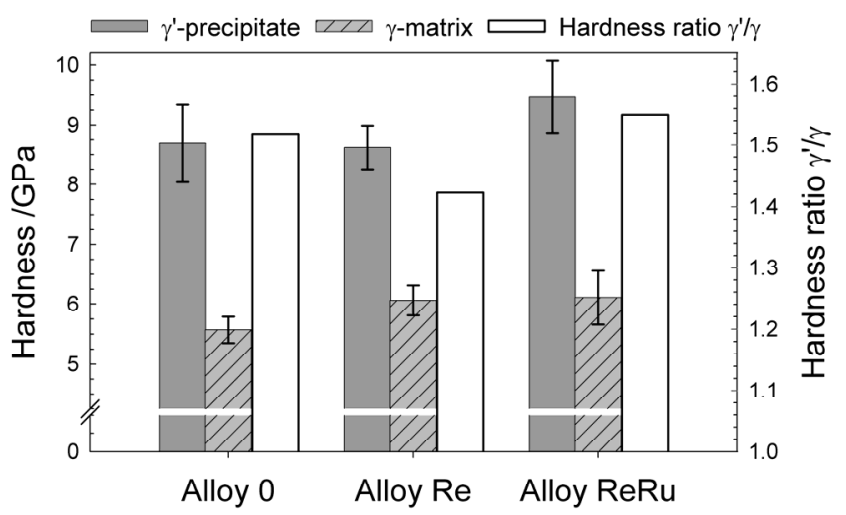

Figure 9: Nanohardness of the phases $\gamma$ and $\gamma$ and $\gamma^{\prime} / \gamma$ hardness ratio measured in Alloy 0, Alloy Re and Alloy ReRu.

The local hardness of the $\gamma$ - and $\gamma^{\prime}$-phase and the respective hardness ratio of $\gamma^{\prime}$ to $\gamma$ for the three alloys investigated in this 
work is plotted in Fig. 9. The reference Alloy 0 exhibits a $\gamma / \gamma$ hardness ratio of about 1.52. By adding 3 wt.\% rhenium the $\gamma^{\prime} / \gamma$ hardness ratio decreases to 1.42 due to the strongly increasing hardness of the $\gamma$-matrix, while no change in $\gamma$-hardness was found. However, the addition of $3 \mathrm{wt} \%$ ruthenium leads to a significant increase in hardness of the $\gamma$-phase, whereas the hardness of the $\gamma$-phase stays almost constant compared to Alloy Re. This means that no strong hardening effect of the $\gamma$-matrix due to ruthenium can be observed and therefore the $\gamma^{\prime} / \gamma$-hardness ratio increases to 1.55 .

\section{Creep Properties and Optimum $\gamma^{\prime}$-Size}

Specimens of Alloy Re with a lattice misfit near zero and Alloy ReRu with a negative lattice misfit at room temperature were creep tested with different initial $\gamma^{\prime}$-sizes between about $0.2 \mu \mathrm{m}$ to $0.6 \mu \mathrm{m}$. The creep curves of all investigated specimens of Alloy ReRu and Alloy Re are shown in Fig. 10 and Fig. 11, respectively.

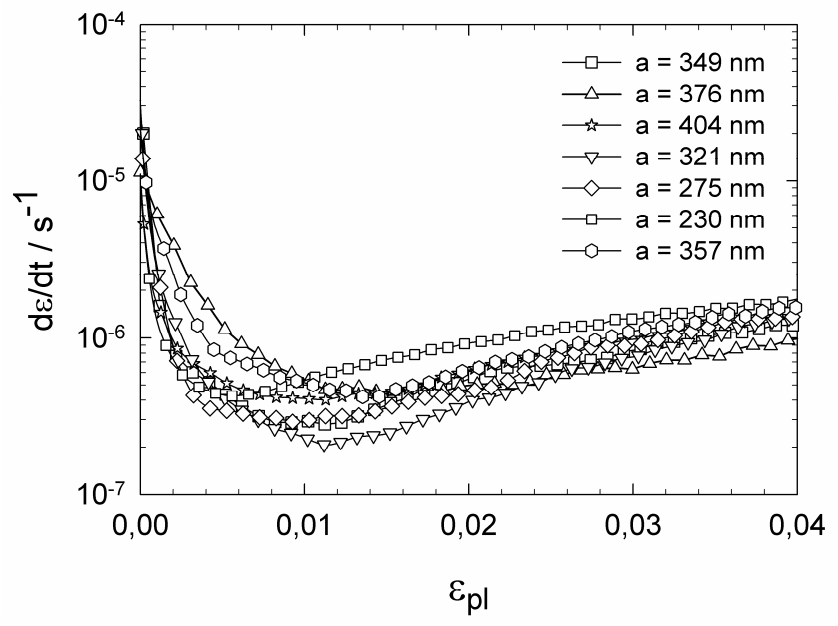

Figure 10: Creep rate versus the plastic creep strain of specimens of Alloy ReRu with different initial $\gamma$-sizes $a$ at $137 \mathrm{MPa}$ at $1100^{\circ} \mathrm{C}$.

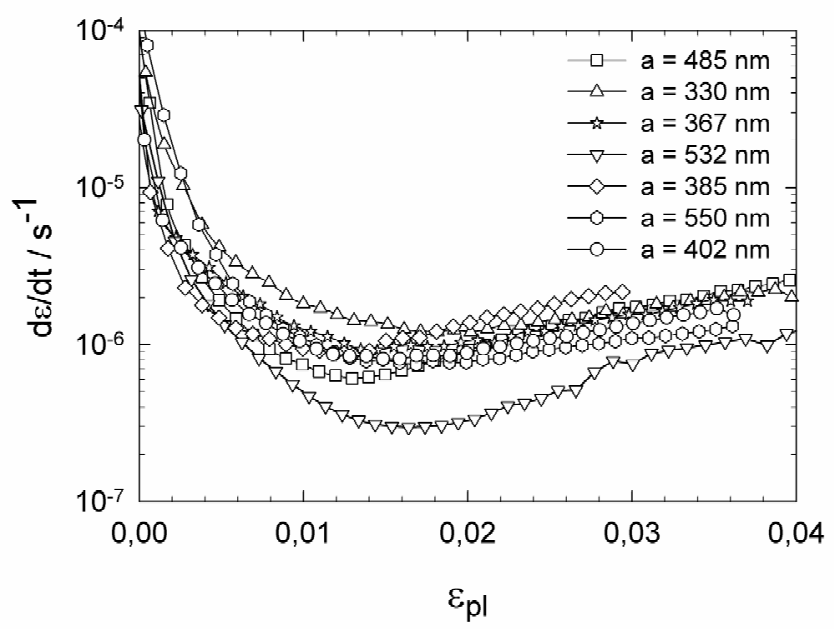

Figure 11: Creep rate versus the plastic creep strain of specimens of Alloy Re with different initial $\gamma^{\prime}$-sizes $a$ at $137 \mathrm{MPa}$ at $1100^{\circ} \mathrm{C}$.
Obviously the creep properties differ significantly for different initial $\gamma^{\prime}$-sizes, which is more clearly visible in Fig. 12 where the minimum creep rate is plotted against the initial $\gamma$-size for the two alloys.

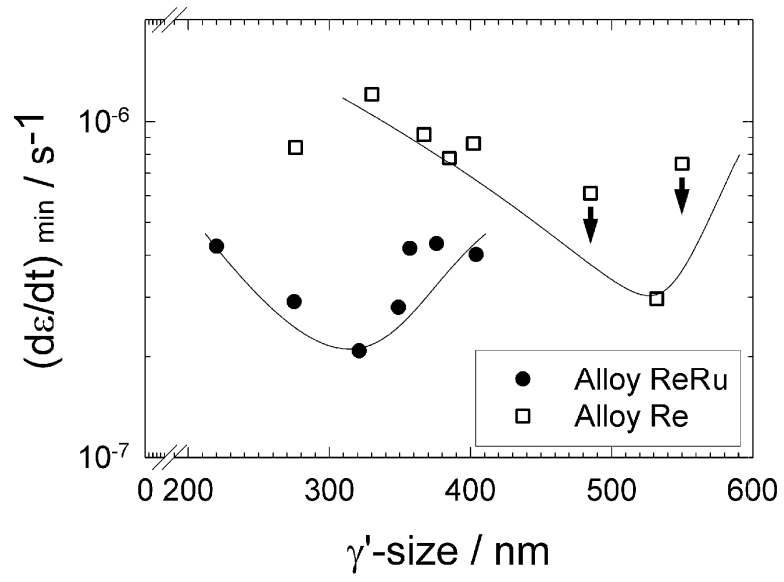

Figure 12: Minimum creep rate in dependence of the initial $\gamma^{\prime}$-size for Alloy ReRu and Alloy Re; two specimens of Alloy Re had a small misoriented columnar grain at the outer diameter of the cylindrical specimen. These misoriented grains probably cause the lower creep strength of these both specimens (marked by arrows).

Both alloys show a maximum creep strength at a specific initial $\gamma$-size. Alloy $\mathrm{ReRu}$ with its negative lattice misfit shows the lowest creep rate of about $2 \times 10^{-7} \mathrm{~s}^{-1}$ for an initial $\gamma^{\prime}$-size of about $320 \mathrm{~nm}$. At a similar $\gamma^{\prime}$-size Alloy Re has a lower creep strength. The minimum creep rate for the optimum initial $\gamma^{\prime}$-size was found to be $3 \times 10^{-7} \mathrm{~s}^{-1}$ in Alloy Re. As shown in Fig. 13 the microstructure for an initial $\gamma^{\prime}$-size of about $330 \mathrm{~nm}$ is different in Alloy ReRu compared to Alloy Re.

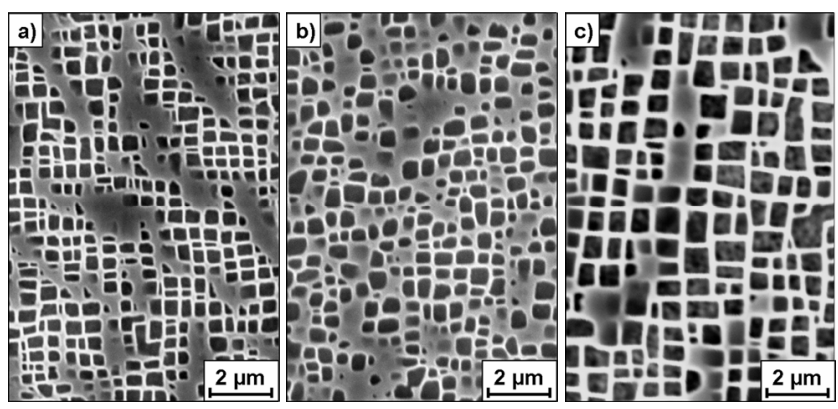

Figure 13: SEM-micrographs of samples of a) Alloy ReRu and b) Alloy Re with similar initial $\gamma$-size of about $320 \mathrm{~nm}$ and of c) Alloy Re with higher initial $\gamma^{\prime}$-size of about $530 \mathrm{~nm}$.

The former alloy possesses cubic precipitates well aligned along $<001>$ directions, while the $\gamma^{\prime}$-precipitates in the latter are more globular and more randomly distributed. With increasing initial $\gamma^{\prime}$ size the minimum creep rate in Alloy Re decreases until a minimum of about $3 \times 10^{-7} \mathrm{~s}^{-1}$ is reached at about $530 \mathrm{~nm}$ initial $\gamma^{\prime}$ precipitate size. At this point the initial $\gamma^{\prime}$-shape became also fully cubic and the precipitates more strongly aligned along $<001>$ as in Alloy ReRu as again is visible in Fig. 13. 
Under the same conditions $\left(137 \mathrm{MPa}\right.$ at $\left.1100^{\circ} \mathrm{C}\right)$ Alloy 0 exhibits a creep rate of about $5 \times 10^{-5} \mathrm{~s}^{-1}$. But due to the bad creep properties of Alloy 0 at that temperature the respective creep curve showed no distinct creep rate minimum and only one specimen was tested.

\section{Discussion}

\section{Partitioning Behavior of Alloying Elements}

The partitioning behavior of alloying elements is an important factor to understand how an alloying element alters the properties of a nickel-base superalloy $[14,17]$. Especially the partitioning behavior of the two elements rhenium and ruthenium is of interest. Rhenium has the most pronounced preference to partition to the $\gamma$-matrix of all elements normally alloyed to nickel-base superalloys $[4,5,6,13,14,15]$. Ruthenium can alter this partitioning behavior of rhenium $[3,6,18]$ and rhenium and ruthenium are actually alloyed to the newest superalloys of the $4^{\text {th }}$ generation $[8,19]$. It will be shown in the subsequent sections that the different results presented in the previous chapter can easily be interpreted in a coherent way if it is considered that reverse partitioning i.e. a more homogenous distribution of rhenium between $\gamma$ - and $\gamma^{\prime}$-phase is present if ruthenium is added. Hence, results regarding such different alloy properties as local hardness, coarsening behavior of the $\gamma$-precipitates and change of lattice misfit and constants support the fact that reverse partitioning was found in EDS-measurements in this work. There is contradictory debate about reverse partitioning in literature. While a number of authors did not find reverse partitioning in ruthenium containing alloys $[4,5,13,15]$ some other works claim that reverse partitioning occurs $[3,18]$. These discrepancies may be caused by the finding that reverse partitioning of rhenium is not only dependent on the ruthenium content but also the presence and content of other alloying elements like chromium and cobalt. There is evidence in literature [6] that low levels of chromium and cobalt promote reverse partitioning. Recently Caroll et al. [6] succeeded in designing ruthenium containing alloys with positive misfit by purposely altering the alloy composition in a way that reverse partitioning occurs. Alloy ReRu investigated here possesses a rather different alloy composition compared to other ruthenium containing alloys in which the absence of reverse partitioning was reported in literature $[4,5,13,15]$. It is assumed that the overall alloy composition of Alloy ReRu promotes the occurrence of reverse partitioning of rhenium. The $\gamma^{\prime}$-volume fraction derived by Blavette's method are in the same range as reported for commercial alloys with similar chemical composition (e.g. CMSX-6) [20].

As nickel-base superalloys are dedicated high temperature materials the partitioning behavior of alloying elements at elevated temperatures is of importance with respect to application. The more homogenous partitioning of ruthenium at higher temperatures means that the ruthenium solid solution strengthening influence on the matrix is reduced at service temperature compared to room temperature. It is an interesting question if this is beneficial or detrimental for the creep properties at very high temperatures, when cutting of $\gamma^{\prime}$-precipitates contribute to the creep deformation [21]. Ruthenium acting as solid solution strengthener in the $\gamma^{\prime}$-precipitates could be beneficial for creep properties because cutting of $\gamma^{\prime}$-particles by dislocations becomes more difficult. On the other hand this means also that a lower content of solid solution strengthening elements in the matrix exists which eases the dislocations moving through the $\gamma$-channels. It should be noted in this respect that actual ruthenium-containing alloys show improvements in creep behavior compared to $2^{\text {nd }}$ and $3^{\text {rd }}$ generation nickel-base superalloys especially at very high temperatures $[8,19,22]$.

The fact, that reverse partitioning of rhenium is not only found in standard heat treated specimens but also in specimens rapidly quenched from $1150{ }^{\circ} \mathrm{C}$ is also interesting in this respect. If more rhenium is alloyed to the $\gamma$-precipitate phase it should also contribute to a higher hardness of the $\gamma^{\prime}$-phase. Accordingly, $\gamma^{\prime}$ cutting in ruthenium containing alloys is not only hindered by the presence of ruthenium as hardening element in the $\gamma^{\prime}$-phase itself but also by a higher amount of rhenium in the $\gamma$-phase if reverse partitioning occurs. That reverse partitioning of rhenium causes a strong hardening of the $\gamma$-phase at least at room temperature will be discussed in more detail in a later section. The occurrence of reverse partitioning would also explain that ruthenium additions improve the phase stability of rhenium containing nickel-base superalloys. Nevertheless, as reverse partitioning is not found in all ruthenium containing alloys it is difficult to explain that improvements in phase stability which are commonly reported for ruthenium containing alloys. Most probably other effects besides reverse partitioning also contribute to the improvement of phase stability in ruthenium containing alloys.

\section{$\underline{\text { Lattice Constants and Misfit }}$}

The changes of lattice constant and lattice misfit in the three alloys can be understood by taking into account the partitioning behavior and especially the effect of reverse partitioning of rhenium in the presence of ruthenium in Alloy ReRu. The change of the lattice misfit in the standard heat treated states from positive values in Alloy 0 to slightly and pronounced negative values in Alloy Re and Alloy ReRu, respectively, is caused by the addition of alloying elements that predominantly partition to the $\gamma$ matrix increasing the lattice constant of this phase and changing the lattice misfit accordingly. This is most obvious by the fact that the lattice misfit in the three alloys shows a nearly linear behavior when plotted vs. the combined element concentration of rhenium, and ruthenium in Fig. 8. As rhenium and ruthenium both partition predominantly to the $\gamma$-matrix the lattice constant of the $\gamma$-phase should increase more than that of the $\gamma$-phase when rhenium and ruthenium are alloyed. Hence, the differences in lattice misfit between the three alloys are directly explained by their alloy composition.

The change of the lattice constants of the $\gamma$ - and $\gamma^{\prime}$-phase is also directly understood by the partitioning of rhenium and ruthenium. In Fig. 14 the lattice constants are plotted versus the content of the elements rhenium, ruthenium and molybdenum measured in the respective phase by TEM-EDS and weighted with Vegard's coefficients found in literature [22, 23, 24]. The refractory elements rhenium, ruthenium and molybdenum are chosen as the concentration of these elements varies the most within both phases in the experimental alloys as shown in Fig. 5. As lattice constants measured in all three alloys linearly depend on the chemical composition of the respective phase it is obvious that all changes of the lattice constants are directly caused and explained by changes in the chemical composition of the respective phase. Comparing Alloy 0 and Alloy Re only the lattice constant of the $\gamma$-matrix is increased by alloying rhenium while the lattice constant of the $\gamma^{\prime}$-phase stays nearly constant. This is due to the fact that rhenium almost predominantly partitions to the $\gamma$-phase and accordingly only affects the lattice constant of this phase. In 
Alloy ReRu the lattice constant of the $\gamma$-phase as well as the lattice constant of the $\gamma^{\prime}$-phase are increased in comparison with Alloy Re and this is caused by the fact that ruthenium does not partition so strongly to the $\gamma$-matrix as rhenium does and hence also increases the lattice constant of the $\gamma^{\prime}$-phase.

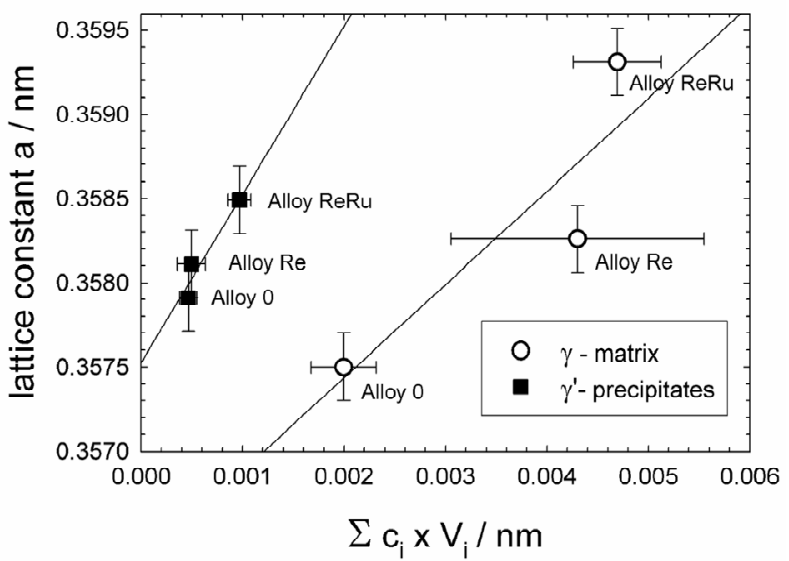

Figure 14: Lattice constants of the phases $\gamma$ and $\gamma$ plotted vs. the chemical composition $c_{i}(i=R e, R u, M o)$ of the respective phase measured by TEM-EDS and weighted with Vegard's coefficient $V_{i}$.

Nevertheless, from Fig. 14, it is obvious that reverse partitioning of rhenium is an important factor to understand the changes in lattice constants between Alloy Re and Alloy ReRu not only qualitatively but quantitatively. Otherwise the increase of the $\gamma^{\prime}$ lattice constant from Alloy Re to Alloy ReRu would not scale with the change in chemical composition of the $\gamma^{\prime}$-phase which includes also an increasing rhenium content due to reverse partitioning.

The shape of the X-ray profiles as well as the misfit changed significantly after creep deformation in Alloy Re and Alloy ReRu, while the changes were minor in Alloy 0. During creep deformation dislocation networks form at the $\gamma / \gamma^{\prime}$-interface. In the initial phase of creep deformation these dislocation networks relax the coherency stresses in one orientation of matrix channels [25]. By directional coarsening a lamellar $\gamma / \gamma^{\prime}$-microstructure forms in which only matrix channels remain where the coherency is decreased by the dislocation networks. When an equilibrium dislocation density is reached so that coherency stresses are fully compensated, further plastic deformation of the matrix channels results in the buildup of an excess dislocation density, which introduces back stresses in the matrix channels. If these back stresses exceed a certain limit it becomes impossible to move new dislocation loops into the matrix channels. Now recovery of dislocation segments stored in the network is necessary before further plastic deformation of the $\gamma$-matrix can occur. This recovery can either happen by glide climb processes of network dislocations and recombination with dislocations from perpendicular matrix channels near the edges of the $\gamma$-precipitates or by cutting of dislocation segments through the $\gamma$-precipitates. With increasing directional coarsening $\gamma^{\prime}$-cutting is the more favorable recovery process.

To understand the results of X-ray measurements in the creep deformed specimens in more detail it is important to remember that the lattice planes under reflection lay perpendicular to the external stress axis. These lattice planes experience the effect of the interfacial dislocation networks to reduce coherency stresses in the beginning and introduce back stresses in the matrix channels afterwards. The coherency stresses in alloys with negative lattice misfit like Alloy $R e$ and Alloy ReRu are compressive in the $\gamma$ - and tensile in the $\gamma$-phase. Hence, the stresses introduced by the dislocation networks should lead to higher lattice constants in the $\gamma$ - and lower lattice constants in the $\gamma^{\prime}$-phase. This is visible as a separation of the $\gamma$ - and $\gamma^{\prime}$-sub peak as observed in the creep deformed specimens of Alloy Re and Alloy ReRu.

How far the $\gamma$ - and $\gamma$-sub peaks separate is dependent on the density of the interfacial dislocation networks. After the onset of recovery processes, either by $\gamma^{\prime}$-cutting or glide climb of dislocation segments near precipitate edges, the dislocation density in the networks should be more or less constant. This is of course under the assumption that the microstructure (matrix channel width, degree of rafting, etc.) does not change significantly. I.e. the separation of the sub peaks is the higher the higher the amount of lattice misfit (leading to a high equilibrium dislocation density) and the harder the $\gamma$-phase (leading to a high excess dislocation density) is if $\gamma^{\prime}$-cutting is the predominant recovery process which is probably the case in directional coarsened $\gamma^{\prime}$-structures. So the finding that the separation of the $\gamma$ and $\gamma$-sub peaks is more pronounced in the creep deformed specimen of Alloy ReRu than in Alloy Re is understandable by the fact that the amount of lattice misfit in Alloy ReRu is higher than in Alloy Re and the $\gamma^{\prime}$-phase in Alloy ReRu is harder than in Alloy Re. Both results stem from room temperature measurements in the standard heat treated states with X-ray diffraction and nanoindentation (these results will be discussed in more detail in the following section). Nevertheless, it is reasonable to assume that the differences in hardness and lattice misfit are still present at creep temperature explaining the higher degree of sub-peak separation in Alloy ReRu.

It is necessary to keep in mind that with increasing temperature the thermal expansion of the $\gamma$-matrix phase is higher than that of the $\gamma$-phase [14] to understand why no significant changes in the shape of the X-ray profile are found in Alloy 0 after creep deformation. If an alloy has already a negative misfit at room temperature the amount of misfit will increase with increasing temperature which leads to higher coherency stresses. This is the reason why differences between the lattice misfits of Alloy ReRu and Alloy Re with their misfit $\delta \leq 0$ at room temperature are also present at high temperature. Alloy 0 with a positive misfit at room temperature could well have a misfit near zero at a temperature of $1100^{\circ} \mathrm{C}$ where the creep experiments were performed. So no coherency stresses are present at creep temperature and at least the portion of the interfacial dislocation network density which was termed as equilibrium density above would be negligible. Hence, no strong shift of the two sub-peaks and accordingly no peak separation is observed in Alloy 0. The assumption that Alloy 0 has a lattice misfit near zero at $1100{ }^{\circ} \mathrm{C}$ is also supported by the shape of the $\gamma$-precipitates in the specimens annealed at that temperature. After $750 \mathrm{~h}$ of annealing no directional coarsening of the $\gamma^{\prime}$-precipitates is observed which would be expected if considerable coherency stresses would be present as driving force at that temperature. Also the globular $\gamma^{\prime}$-precipitates found in the standard heat treated state of Alloy 0 support this interpretation. If the lattice misfit is near zero at higher temperatures in Alloy 0 also no driving force due to coherency stresses exist to form cubic $\gamma^{\prime}$-precipitates at the temperature of the standard-heat treatment. The cubic precipitates in Alloy Re and Alloy $R e R u$ which are especially well developed and aligned in Alloy ReRu give hints 
that these alloys have a significant amount of lattice misfit at the temperature of the standard heat treatment. This is in accordance with the fact that interfacial dislocation networks form in these two alloys during creep.

\section{Local Hardness of $\gamma$ - and $\gamma$-Phase}

Nanoindentation in the AFM is to the knowledge of the authors the only available method to investigate alloying effects on the mechanical properties of single phases in nano-scaled materials as nickel-base superalloys are [26].

In comparison to Vicker's hardness measurements the hardness measured in this work is slightly higher due to the indentation size effect. For details see $[26,27,28]$. As expected and also found in previous works $[26,29]$ the hardness of the intermetallic $\gamma$-phase in the three investigated alloys is always higher than the $\gamma$-matrix phase. Rhenium which is known to be a potent solid solution strengthener in nickel-base superalloys [16] was found to be nearly exclusively enriched in the $\gamma$-matrix in Alloy Re. This leads to a significant increase in the hardness of the $\gamma$-matrix compared with Alloy 0. This effect showing the solid solution strengthening potential of rhenium was also found by Durst et al [29]. In a similar manner as the differences in lattice constants of the phases $\gamma$ and $\gamma$ also the changes in hardness of the $\gamma$ - and $\gamma^{\prime}$-phase in Alloy ReRu compared with Alloy Re can only be understood by the effect of reverse partitioning. Otherwise it would be expected that the hardness of the $\gamma$-matrix increases more than the hardness of the $\gamma$-phase as ruthenium partitions more strongly to the $\gamma$ phase. The finding that the hardness of the $\gamma^{\prime}$-phase increases significantly while the hardness of the $\gamma$-matrix stays nearly unchanged is explained by the decrease of rhenium and also to a lesser extent molybdenum in the $\gamma$-matrix due to reverse partitioning which compensates nearly fully the hardness increase caused by ruthenium in the $\gamma$-phase. Also rhenium, which is now redistributed to the $\gamma$-phase causes a very strong increase in hardness there. This interpretation is supported by plotting the hardness of both phases vs. the lattice constant measured by X-ray diffraction as shown in Fig. 15.

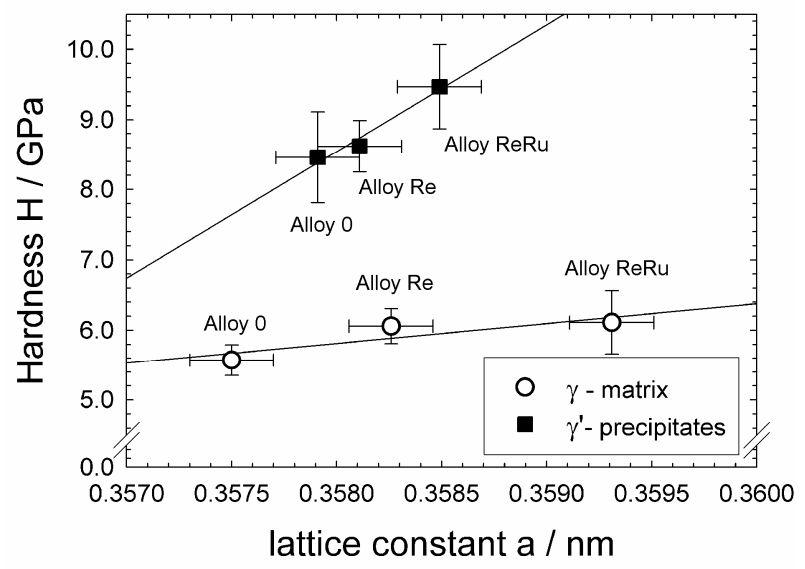

Figure 15: Hardness of the phases $\gamma$ and $\gamma^{\prime}$ plotted versus the lattice constant of the respective phase.

As the hardness depends linearly on the lattice constant (Fig. 15) and the lattice constant itself depends linearly on the element concentration (Fig. 14) alloying elements seem to change the lattice constant and hardness in the same way. Most probably the hardness increase is caused by solid solution strengthening due to the paraelastic interaction. This means that an element changes the local lattice constant in its vicinity.

From hardness measurements at room temperature of course only indirect conclusions could be made on the mechanical properties at service temperature. Nevertheless, while nanoindentation can directly measure mechanical properties in single phases only at low temperatures other methods have also their drawbacks. Alternative indirect methods like creep testing of special alloy compositions reassembling the chemical composition of one of the two phases are handicapped by experimental difficulties like exactly determining the composition of the phases at elevated temperature or avoiding the presence of small amounts of the second phase in those alloys.

From the nanoindentation results presented in this work it can clearly be stated that rhenium has a strong hardening effect on both phases, $\gamma$ and $\gamma$. The effects of ruthenium on the hardness of the $\gamma$-matrix cannot be determined clearly as the lower content of rhenium due to reverse partitioning with the corresponding effects on hardness overshadows the effects of ruthenium. In the $\gamma^{\prime}$-phase a strong increase of hardness due to ruthenium addition is found. By relating the results from nanohardness measurements to the changes in lattice constants in the three alloys as shown in Fig. 15 it can be deduced from the linear relationship that solid solution hardening by paraelastic interaction is the effect by which rhenium and ruthenium influence the mechanical properties of $\gamma$ and $\gamma$. This is of special interest as this effect should also be present at elevated temperature. From the partitioning behavior at elevated temperature and here especially the tendency of ruthenium to distribute more homogenously between $\gamma$-matrix and $\gamma^{\prime}$-phase it is quite probable that the hardening of the $\gamma^{\prime}$ precipitates by ruthenium is even more pronounced at higher temperature.

A decreased susceptibility for $\gamma$-cutting due to a higher hardness of the $\gamma^{\prime}$-phase would lead to a higher density of interfacial dislocation networks due to a higher amount of excess dislocations. Some authors claim that dense dislocation networks formed in ruthenium containing nickel-base superalloys are the main contribution to the excellent creep properties of these alloys $[7,19]$.

\section{$\underline{\text { Microstructural Development at High Temperature }}$}

Alloying elements which affect diffusion influence the coarsening of $\gamma^{\prime}$-precipitates by this. But of course if an alloying element is more or less homogenously distributed between $\gamma$-matrix and $\gamma$ precipitates or tends to enrich in one of the two phases also plays a role. While $\gamma$-particles grow the local chemical composition has to be adjusted so that elements which are present in a higher concentration in the $\gamma$-matrix than in the $\gamma^{\prime}$-phase are removed by diffusion. So if an element is strongly enriched in the $\gamma$-matrix high amounts of it have to be removed by diffusion before a local composition is reached which allows the $\gamma^{\prime}$-precipitate to grow further. A prominent example of this effect is the so-called bowwave of rhenium reported in literature in front of moving $\gamma^{\prime}$ interfaces [30]. Hence, elements like rhenium with a pronounced tendency to enrich in one of the two phases and a slow diffusion speed strongly control the kinetics of $\gamma^{\prime}$-coarsening in nickel-base superalloys.

The results presented in this work for the precipitate coarsening during short time annealing show that the growth of the 
precipitates is diffusion controlled as the relationship between $(a / 2)^{3}$ and the annealing time for the three alloys under investigation is linear [31]. Hence, the significant deceleration of the particle growth in the rhenium containing Alloy Re and Alloy $R e R u$ is caused by the slowdown of diffusion processes caused by rhenium. It is surprising that the difference in the coarsening speed of the $\gamma^{\prime}$-particles is so small when comparing Alloy Re and Alloy ReRu. That the effect of ruthenium addition on the coarsening behavior is not pronounced is probably caused by the fact that at $1100{ }^{\circ} \mathrm{C}$ where the annealing tests were performed ruthenium is less strongly enriched in the $\gamma$-matrix than at room temperature as was found in the EDS-measurements in rapidly quenched specimens. So it is not necessary to transport significant amounts of ruthenium away by diffusion to allow movement of the $\gamma / \gamma$-interfaces during particle growth. Reverse partitioning in Alloy ReRu also reduces the amount of rhenium necessary to be transported away before the $\gamma$-precipitates can grew further which also contributes to the effect, that ruthenium addition not strongly slows down particle coarsening in Alloy ReRu compared with Alloy Re.

\section{Creep Properties and Optimum $\gamma^{\prime}$-Size}

$4^{\text {th }}$ generation nickel-base superalloys contain a high amount of refractory alloying elements and typically exhibit high negative $\gamma / \gamma^{\prime}$-lattice misfit $[7,19,8,22]$. As reported by Nathal in a previous work [32] the creep strength at elevated temperature depends on the initial $\gamma^{\prime}$-size. Thus it is interesting to investigate which $\gamma^{\prime}$-particle sizes are desirable in alloys possessing a high lattice misfit with respect to creep strength. The investigations of Nathal were performed on alloys possessing different lattice misfits of $-0.1 \%$ and $-0.17 \%$. The magnitude of lattice misfit was gained by measuring specimens which were aged until loosing coherency between $\gamma$ and $\gamma^{\prime}$. In the present work Alloy ReRu with a lattice misfit of about $-0.22 \%$ even in the standard heat treated state was compared to Alloy Re with a lattice misfit around zero. In agreement with literature [32] it was found that a strong dependence of the minimum creep rate on the initial $\gamma$-size exists and that the higher the lattice misfit the smaller the initial $\gamma^{\prime}$-size has to be to achieve maximum creep strength. Due to a higher constrained lattice misfit of Alloy ReRu even in the standard heat treated state the optimum initial $\gamma^{\prime}$-size is shifted to lower values of about $320 \mathrm{~nm}$ compared to Alloy Re with about $530 \mathrm{~nm}$. It is assumed that the reason for this behavior is the same as proposed already by Nathal and lies in the initial microstructure. Generally, very small $\gamma$-precipitates exhibit a globular shape and are not well aligned. With increasing $\gamma^{\prime}$-size the $\gamma^{\prime}$-precipitates become more and more cubic as this precipitate shape is favorable with respect to minimizing the coherency stresses. As Alloy ReRu possesses higher coherency stresses based on the higher constrained lattice misfit the $\gamma$-shape is fully cubic and the precipitates are wellaligned along $<001>$ at lower $\gamma$-size (Fig. 13). Nathal concludes that these strongly aligned cubic precipitates lead to a more orderly precipitate structure during directional coarsening with a lower amount of terminations of the $\gamma$-rafts per unit area which enhance the creep resistance. This would explain why specimens with an optimum $\gamma^{\prime}$-size and cubic well aligned precipitates have a better creep resistance compared with specimens with precipitate sizes below the optimum $\gamma^{\prime}$-size. Nevertheless, this view cannot be fully supported based on the results presented in this work as the creep rates at higher plastic strains (i.e. when the rafted microstructure should be fully developed) show neither the strong dependence on the initial $\gamma^{\prime}$-sizes as the minimum creep rates nor does a specimen with a lower minimum creep rate necessarily also possess a lower creep rate at higher plastic strains (see Fig. 10 and Fig. 11). At $\gamma^{\prime}$-sizes greater than the optimum $\gamma^{\prime}$-size the creep strength also decreases. One reason may be that the channel width of the $\gamma$-matrix is also increased which leads to lower Orowan stresses. Also, the fraction of $\gamma / \gamma^{\prime}$-interfaces is lower in a coarsened microstructure. Hence, dislocations gliding through matrix channels less often interact with interfacial dislocation networks. Assuming that these interfacial dislocations hinder dislocation movement through the matrix channels and act as barrier against cutting of the directionally coarsened $\gamma^{\prime}$ precipitates, the creep strength decreases [7, 19]. As already mentioned above it is not visible from the creep data at higher plastic strains where hardening effects due to interfacial dislocation networks should play a role that the initial $\gamma$-size influences the creep behavior at this later stages of creep as significantly as the minimum creep rate.

Another interesting result of the creep experiments is the difference in the overall minimum creep rate of Alloy Re with $3 \times 10^{-7} \mathrm{~s}^{-1}$ compared to $2 \times 10^{-7} \mathrm{~s}^{-1}$ of Alloy ReRu. Nevertheless, it is open if this difference is caused by strengthening of the matrix phase in Alloy ReRu due to additional ruthenium, by an increase in $\gamma^{\prime}$-hardness due to reverse partitioning or by other more indirect effects as slower $\gamma^{\prime}$-particle coarsening, differences in $\gamma^{\prime}$-volume fraction etc.

\section{Conclusions}

Three experimental nickel-base superalloys with varying contents of rhenium and ruthenium were investigated to understand the influence of these alloying elements on the alloy properties. A number of different methods were employed as conventional microstructure analysis using TEM and SEM, analytical electron microscopy, X-ray diffraction and nanoindentation in an AFM. Results on the local hardness of the single phases $\gamma$ and $\gamma^{\prime}$ in ruthenium containing alloys are to the knowledge of the authors not available anywhere else in literature until now.

The most important findings are as follows:

- Rhenium of all alloying elements partitions most strongly to the $\gamma$-matrix. Nevertheless, in the presence of ruthenium rhenium is more homogenously distributed between the $\gamma$ - and $\gamma^{\prime}$-phase. This partitioning behaviour and reverse partitioning is also found at higher temperature.

- Ruthenium is slightly enriched in the $\gamma$-matrix but homogenizes between $\gamma$ - and $\gamma^{\prime}$-phase with increasing temperature.

- Rhenium additions increase the lattice constant of the $\gamma$ matrix, while ruthenium additions increase the lattice constants of the $\gamma$ - and $\gamma$-phase also due to reverse partitioning of rhenium.

- The hardness of the $\gamma$-matrix increases when rhenium is alloyed. Alloying of ruthenium increases the hardness of the $\gamma$-precipitates but no change in the $\gamma$-hardness was found most probably due to reverse partitioning. Both elements probably influence the hardness of the respective phases by solid solution hardening.

- The creep properties of the alloys are influenced by the initial $\gamma^{\prime}$-size. If rhenium and ruthenium are present the amount of lattice misfit was found to be higher and the optimum $\gamma^{\prime}$-size with respect to creep properties was smaller due to more cubic and better aligned $\gamma^{\prime}$-precipitates already present at that $\gamma^{\prime}$ - 
size. Also denser interfacial dislocation networks form in crept specimens if ruthenium is alloyed. This is probably a combined effect of the higher amount of lattice misfit and higher $\gamma$-hardness.

\section{Acknowledgement}

The work presented in this paper was financially supported by the German Science Foundation (DFG) in the frame of the DFG graduate school 1229. The authors thank the Institute of Science and Technology of Metals of the University Erlangen-Nürnberg for providing access to their Bridgman casting unit.

\section{References}

[1] E. W. Ross, C. T. Sims, "Nickel-Base Alloys", Superalloys II - High temperature materials for aerospace and industrial power, eds. C. T. Sims et al. (New York, NY: John Wiley \& Sons, Inc.,1987), 97-133.

[2] T. Kitashima, D. H. Ping , H. Harada, T. Kobayashi, "Atom probe microanalysis of fifth-generation nickel-base single crystal superalloys", Materials for Advanced Power Engineering 2006, Proc. of the $8^{\text {th }}$ Liège Conference, Part I, eds. J. Lecomte-Beckers et al. (Jülich, Germany: Schriften des Forschungszentrums Jülich, Energy Technology, 2006), 495-506.

[3] K. S. O'Hara, W. S. Walston, E. W. Ross, R. Darolia, US Patent No. 5,482,789, General Electric Company, Cincinnati, $\mathrm{OH}$ and Lynn, MA, 1996.

[4] R. Bürgel, J. Grossmann, O. Lüsebrink, H. Mughrabi, F. Pyczak, R. F. Singer, A. Volek, "Development of a new alloy for directional solidification of large industrial gas turbine blades", Proc. 10th Int. Symposium on Superalloys, Superalloys 2004, eds. K. A Green et al. (Warrendale, PA: The Minerals, Metals and Materials Society, 2004), 25-34.

[5] A. Volek, F. Pyczak, R. F. Singer, H. Mughrabi, "Partitioning of Re between $\gamma$ and $\gamma$ phase in nickel-base superalloys", Scripta Mat., 52 (2005), 141-145.

[6] L.J. Carroll, Q. Feng, J.F. Mansfield, T.M. Pollock, "High Refractory, Low Misfit Ru-Containing Single-Crystal Superalloys", Met. Mat. Trans., 37A (2006), 2927-2938.

[7] J. X. Zhang, T. Murakumo, H. Harada, Y. Koizumi, T. Kobayashi, "Creep deformation mechanisms in some modern single-crystal superalloys", Proc. 10th Int. Symposium on Superalloys, Superalloys 2004, eds. K. A Green et al. (Warrendale, PA: The Minerals, Metals and Materials Society, 2004), 189-195.

[8] A. C. Yeh, C. M. F. Rae, S. Tin, "High temperature creep of Ru-bearing Ni-base single crystal superalloys", Proc. 10th Int. Symposium on Superalloys, Superalloys 2004, eds. K. A Green et al. (Warrendale, PA: The Minerals, Metals and Materials Society, 2004), 677-685.

[9] M. Wilkens, K. Eckert, "Röntgenographische Untersuchungen über die Versetzungsanordnung in plastisch verformten Kupfereinkristallen”, Z. Naturf., 19a (1964), 459470.

[10] http://www.esrf.eu/computing/scientific/xop2.1/
[11] W. C. Oliver, G. M. Pharr, "An improved technique for determining hardness and elastic modulus using load and displacement sensing indentation experiments", J. Mater. Res., 7 (1992), 1564-1583

[12] U. Hemmersmeier, M. Feller-Kniepmeier, "Element distribution in the macro- and microstructure of nickel base superalloy CMSX-4”, Mater. Sci. Eng. A248 (1998), 87-97.

[13] T. Yokokawa, M. Osawa, K. Nishida, T. Kobayashi, Y. Koizumi, H. Harada, "Partitioning behavior of platinum group metals on the $\gamma$ and $\gamma$ phases of Ni-base superalloy at high temperatures", Scripta Mat., 49 (2003), 1041-1046.

[14] F. Pyczak, B. Devrient, H. Mughrabi, "The effects of different alloying elements on the thermal expansion coefficients, lattice constants and misfit of nickel-based superalloys investigated by X-ray diffraction”, Proc. 10th Int. Symposium on Superalloys, Superalloys 2004, eds. K. A Green et al. (Warrendale, PA: The Minerals, Metals and Materials Society, 2004), 827-836.

[15] R. C. Reed, A. C. Yeh, S. Tin, S. S. Babu, M. K. Miller, "Identification of the partitioning characteristics of ruthenium in single crystal superalloys using atom probe tomography", Scripta Mat., 51 (2004), 327-331.

[16] D. Blavette, P. Caron, T. Khan, "An atom-probe study of some fine-scale microstructural features in Ni-based single crystal superalloys", Proc. 6th Int. Symposium on Superalloys, Superalloys 1988, eds. S. Reichmann et al., (Warrendale, PA: The Metallurgical Society of AIME, 1988), 305-314.

[17] F. Pyczak, B. Devrient, F. C. Neuner, H. Mughrabi, "The influence of different alloying elements on the development of the $\gamma / \gamma^{\prime}$-microstructure of nickel-base superalloys during high temperature annealing and deformation", Acta Mat., 53 (2005), 3879-3891.

[18] A. P. Ofori, C. J. Humphreys, S. Tin, C. N. Jones “A TEM study of the effect of platinum group metals in advanced single crystal nickel-base superalloys", Proc. 10th Int. Symposium on Superalloys, Superalloys 2004, eds. K. A Green et al. (Warrendale, PA: The Minerals, Metals and Materials Society, 2004), 787-794.

[19] A. Sato, A. C. Yeh, T. Kobayashi, T. Yokokawa, H. Harada, T. Murakumo, J. Zhang, "A $5^{\text {th }}$ generation Ni-base single crystal superalloy with superior elevated temperature properties", Materials for Advanced Power Engineering 2006, Proc. of the $8^{\text {th }}$ Liège Conference, Part I, eds. J. Lecomte-Beckers et al. (Jülich, Germany: Schriften des Forschungszentrums Jülich, Energy Technology, 2006), 287298.

[20] H. Frenz, J. Kinder, H. Klingelhöfer, P. D. Portella, "Behavior of single crystal superalloys under cyclic loading at high temperatures", Proc. $8^{\text {th }}$ Int. Symposium on Superalloys, Superalloys 1996, eds. R. D. Kissinger et al., (Warrendale, PA: T The Minerals, Metals and Materials Society, 1996), 305-312.

[21] R. Srinivasan, G. F. Eggeler, M. J. Mills, " $\gamma$ '-cutting as ratecontrolling recovery process during high-temperature and low-stress creep of superalloy single crystals", Acta Mat., 48 (2000), 4867-4878. 
[22] P. Caron, "High $\gamma^{\prime}$ solvus new generation nickel-based superalloys for single crystal turbine blade applications", Proc. $9^{\text {th }}$ Int. Symposium on Superalloys, Superalloys 2000, eds. T. M. Pollock et al. (Warrendale, PA: The Minerals, Metals and Materials Society, 2000), 737-746.

[23] Y. Mishima, S. Ochiai, T. Suzuki, "Lattice Parameters of $\mathrm{Ni}(\gamma) \mathrm{Ni}_{3} \mathrm{Al}\left(\gamma^{\prime}\right)$ and $\mathrm{Ni}_{3} \mathrm{Ga}\left(\gamma^{\prime}\right)$ Solid Solutions with Additions of Transition and B-subgroup Elements", Acta Metall., 33 (6) (1985), 1161-1169.

[24] W. Pearson, A Handbook of Lattice Spacings and Structures of Metals and Alloys, vol. 2 (Oxford, UK: Pergamon Press, 1967), 1135.

[25] A. Lasalmonie, J. L. Strudel, "Interfacial dislocation networks around $\gamma^{\prime}$ precipitates in nickel-base alloys", Phil. Mag., 32, no. 5 (1975), 937-949.

[26] M. Göken, M. Kempf, "Microstructural properties of superalloys investigated by nanoindentation in an atomic force microscope", Acta Mat., 47, No. 3, (1999), 1043-1052.

[27] Q. Ma, D. R. Clarke, "Size dependant hardness in silver single crystals", J. Mat. Res., 10, (1995), 853-863

[28] K. Durst, B. Backes, O. Franke, M. Göken, "Indentation size effect in metallic materials: Modeling strength from pop-in to macroscopic hardness using geometrically necessary dislocations”, Acta Mat., 54 (2006), 2547-2555.

[29] K. Durst, M. Göken, "Micromechanical characterisation of the influence of rhenium on the mechanical properties in nickel-base superalloys”, Mat. Sci. Eng., A 387-389 (2004), 312-316.

[30] P. J. Warren, "An atom probe study of the distribution of rhenium in a nickel-based superalloy", Mat. Sci. Eng., A 250 (1998), 88-92.

[31] R. A. MacKay, M. V. Nathal, “ $\gamma$ ' Coarsening in High Volume Fraction Nickel-Base Alloys", Acta Met. Mat., 41 (1990), 993-1005.

[32] M. V. Nathal, "Effect of Initial Gamma Prime Size on the Elevated Temperature Creep Properties of Single Crystal Nickel Base Superalloys", Met. Trans. A, 18A (1987), 19871961. 